\title{
The Imaging Research for Thoracolumbar Spine Lateral Vascular Anatomy
}

\section{Hou-Gan Ou Yang*}

Anatomy Education and Research Institute, Jiangxi University of Traditional Chinese Medicine, Nanchang, China

\begin{abstract}
Object: To provide the anatomic basis of imaging for the segmental vessels of the thoracolumbar spine side and measure the distance between two adjacent segmental arteries in the three-dimensional images.
\end{abstract}

Method: After conducting angiography for 50 cases of normal adults through the ulnar vein, carrying out thoracolumbar CT scans. In the image observation: The course and adjacent relationship of the segmental vessels; Measuring range: the distance between the upper and lower segmental arteries of the middle line of spine side.

Results: We could observe the course and the adjacent relationship of thoracolumbar spine lateral vascular. In between the upper and lower segmental vessels consists a relatively non-vascular nerve safe zone where the intervertebral space locates. And we surveyed the related datas.

Conclusion: Three-dimensional reconstruction of CT images can effectively observe the course and adjacent relationship of the thoracolumbar spinal lateral segmental artery, and can accurately measure the safe zone.

Keywords: Image; Segmental artery; Safe zone

\section{Introduction}

Thoracolumbar spine section refers to the $\mathrm{T}_{11}-\mathrm{L}_{2}$ section, which is an important structural transition zone at the junction of thoracolumbar. This section has a deeper anterior approach position, complex anatomical structure, and high risk for larger trauma and complications during traditional surgeries.

Since Mack applied video-assisted thoracoscopic surgery (VATS) to treat thoracic disc herniation for the first time in 1993, it has been widely used in the treatment of various thoracic and lumbar spine diseases. Compared with the conventional open surgery, it has following advantages: less traumas, less bleeding and faster recovery, less interference to the old and weak patients, or those with poor heart and lung function, extending the surgical indications. However, one of the keys to the VATS is how to properly deal with the segment blood vessels. But at present, few applications- oriented anatomical studies have been done on thoracolumbar lateral vascular, especially those combined with image technology.

This study focuses on the research of the arrangement, distribution and the adjacent relationship of the thoracolumbar spinal lateral vascular by image technology. And study result proves that the threedimensional CT image can be utilized as a reference for video-assisted thoracoscopic surgery (VATS) plan.

\section{Materials and Methods}

\section{Materials}

Materials in this study include the CT three-dimensional images of 50 normal adults without thoracic or lumbar diseases (female $=24$, male $=26$, height $153-175 \mathrm{~cm}$, female's average height was $160 \mathrm{~cm}$ and male's average height was $170 \mathrm{~cm}$, average age was 41 years old). All the images were provided by the People's Liberation Army 169 Hospital Image Department.

\section{Methods}

Methods in this study include image data processing. The following are the procedures:

1. Choosing 50 normal adults without thoracic and lumbar diseases (all of these cases were healthy volunteers, CT scanning for no other reason. We obtained the approval of Ethics Committee, and each volunteer knew the facts of the case and agreed.);

2. Conducting CT scan after opacification and the threedimensional reconstruction at CT workstation;

3. Observing the arrangement of the segmental blood vessels;

4. Measuring the distance between two adjacent segmental arteries at the mean line of the lateral thoracolumbar spine (Figures 1 and 2).

\section{Statistical methods}

Comparisons between the left and right were performed using paired sample $t$ test and the independent sample $t$ test in terms of gender. Statistics are performed by using the SPSS for Windows software package (version 12, SPSS Inc., Chicago) and probabilities of $\mathrm{p}<0.05$ were accepted to show statistical significance.

\section{Results}

Observing the three-dimensional images also provides the information about the arrangement and distribution of segmental vessels. The segmental arteries constantly exist in the supersulcus of the corresponding vertebral center with occasional absence $(2 / 100)$. The segmental arteries originate from the posterior wall of aorta, having a lower starting position than the corresponding vertebral height, and running backward and upward with the corresponding vertebral

*Corresponding author: Hou-Gan OuYang, Anatomy Education and Research Institute, Jiangxi University of Traditional Chinese Medicine, Nanchang, China, Tel: 13755618683; Fax: 0791-87118907; E-mail: 730030659@qq.com

Received November 02, 2012; Accepted November 26, 2012; Published November 28, 2012

Citation: Ou Yang HG (2013) The Imaging Research for Thoracolumbar Spine Lateral Vascular Anatomy. J Spine 2:127. doi:10.4172/2165-7939.1000127

Copyright: ( 2013 Ou Yang HG. This is an open-access article distributed under the terms of the Creative Commons Attribution License, which permits unrestricted use, distribution, and reproduction in any medium, provided the original author and source are credited. 
body, then to the central supersulcus of the corresponding vertebral body's side. Segmental arteries have almost equal distance to the upper and lower discs. Many of bilateral same sequence segmental arteries' starting points are in the same horizontal plane, and are bilaterally symmetric (49/50); it is hard to see the segmental arteries' branches on the side of the vertebral body in images. Reconstructed image can show the relationship between the artery and the vertebral body, but cannot show the organizations such as the nervus splanchnicus major, hemiazygos vein, and sympathetic trunk (Figures 1 and 2).

In the midline of the vertebral body side, the distance between adjacent segmental arteries in the $\mathrm{T}_{10 / 11}, \mathrm{~T}_{11 / 12}, \mathrm{~T}_{12} / \mathrm{L}_{1}, \mathrm{~L}_{1 / 2}$, and $\mathrm{L}_{2 / 3}$ gradually increases (Table 1$)$ for the left and right. That is $(23.37 \pm$ $0.98),(23.37 \pm 0.98),(29.06 \pm 1.33),(32.66 \pm 1.23),(33.52 \pm 1.40) \mathrm{mm}$ and $(23.40 \pm 1.28),(25.59 \pm 2.01),(29.13 \pm 2.10),(32.43 \pm 1.18),(33.31$

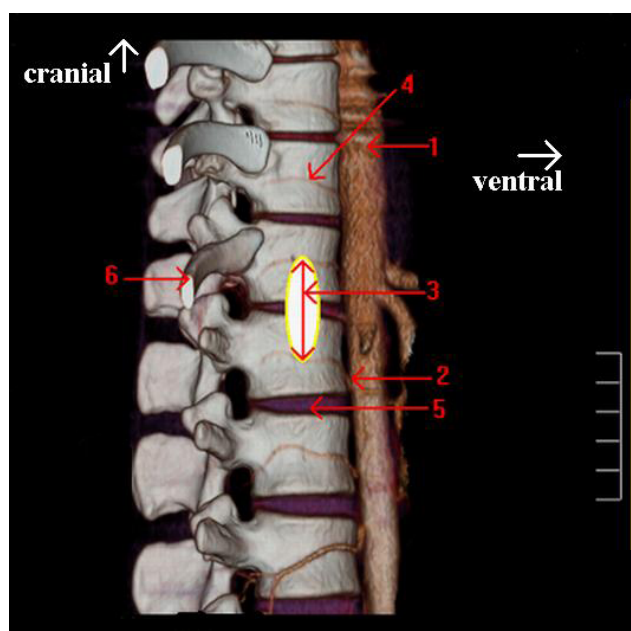

1) Aorta; 2) The starting point of segmental arteries; 3) The distance between both segmental arteries; 4) Segmental artery; 5) Intervertebral place; 6) $T_{12}$ rib

Figure 1: Right view: Measuring the distance between two adjacent segmental arteries at the mean line of the lateral thoracolumbar spine.

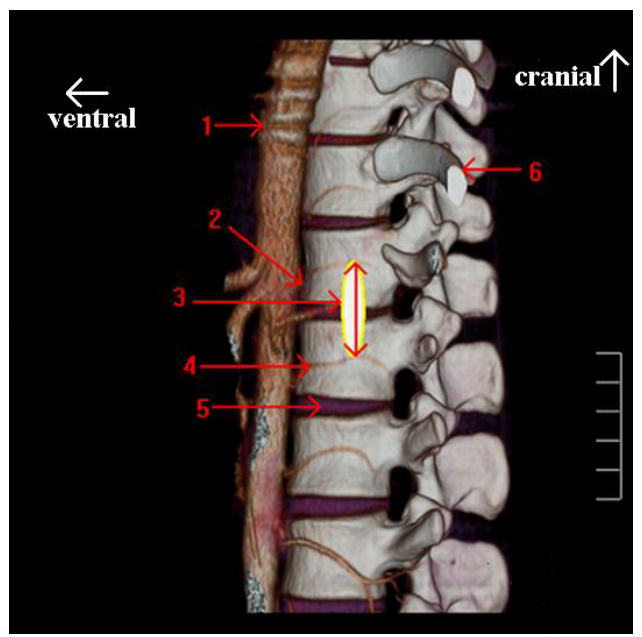

1) Aorta; 2) The starting point of segmental arteries; 3) The distance between both segmental arteries; 4) Segmental artery; 5) Intervertebral place; 6) $T_{12}$ rib

Figure 2: Left view: Measuring the distance between two adjacent segmental arteries at the mean line of the lateral thoracolumbar spine.

\begin{tabular}{|cccc|}
\hline Items & Left & Right & Average \\
\hline T10-T11 & $23.37 \pm 0.98$ & $23.40 \pm 1.28$ & $23.39 \pm 1.08$ \\
T11-T12 & $25.56 \pm 1.85$ & $25.59 \pm 2.01$ & $25.57 \pm 1.95$ \\
T12-L1 & $29.06 \pm 1.33$ & $29.13 \pm 2.10$ & $29.10 \pm 1.70$ \\
L1-L2 & $32.66 \pm 1.23$ & $32.43 \pm 1.18$ & $32.50 \pm 1.28$ \\
L2-L3 & $33.52 \pm 1.40$ & $33.31 \pm 1.27$ & $33.41 \pm 1.34$ \\
\hline
\end{tabular}

Table 1: The distance between segmental arteries $(x \pm s \mathrm{~mm})$.

$\pm 1.27) \mathrm{mm}$ respectively. The blood vessel space increases gradually from top to bottom.

\section{Discussions}

The significance of conducting the segmental blood vessel anatomy research

The video-assisted thoracoscopic thoracolumbar anterior approach is a new minimally invasive technique [1]. The indications for VATS are: interbody fusion, prolapse of intervertebral disc, vertebral tuberculosis, vertebral tumors, vertebral fractures, scoliosis, etc [2]. Properly dealing with the segmental artery is one of the keys to the success of the video-assisted thoracoscopic surgery [3]. The segmental artery, which directly originates from the aorta, has high blood vessel pressure, and bleeds more after injury. It is even more difficult to be controlled when bleeding.

At present, the study has been reported in the literature on the spinal cord anemic damage occurring after blocking the anterolateral segmental artery in the anterior approach and the long-term impact of bone fusion.

Yuan et al. [4] studies suggested that, with the increasing number of segmental blood vessel ligation, the risk of the corresponding spinal cord ischemia would be higher. Li Wei-guo [5], according to animal experiment, indicated that the ligation of segmental blood vessels would affect the anterior approach bone fusion, reduce the hardness of bone fused, and have an obvious impact on the occurrence of the intervertebral disc degeneration. It was also reported that the risk of having the spinal cord ischemic damage caused by blocking many segmental blood vessels would greatly increase [6].

The anterior radicular artery is the branch of the dorsal branch. The most important branch of anterior radicular artery is the Adamkiewicz artery, which is the important source of the thoracolumbar spinal cord blood supply. If damaged in surgery, it will possibly lead to the spinal anterior artery syndrome and at last cause paraplegia [7]. The results of the Adamkiewicz artery's autopsy by Koshino [8] showed that $72 \%$ of the Adamkiewicz arteries originated from the left intercostal arteries or lumbar arteries, $91 \%$ in the $\mathrm{T}_{8}-\mathrm{L}_{1}$ level. So, we should make full preparation during surgery for Adamkiewicz artery protection.

In a word, in order to adapt to the development of video-assisted thoracoscopic surgery, it is necessary to conduct the researches on this section vascular. Therefore more specific anatomy researches of this field are needed for clinical application.

However, Few scholars have conducted specific research on the thoracolumbar spine lateral segmental vascular. Moreover, the study on the related image of segmental vascular is extremely inadequate.

Based on these conditions, we have made the study on the related image of segmental vascular.

\section{Influence of safe zone to anterior approach}

We believe that it is entirely possible to have an operation (such as interbody fusion and disk replacement) on the zone between 
the adjacent segmental arteries without the need of the ligation for segmental arteries [9]. In terms of the characteristics that the segmental vein is thin and bleeds easily after stretched, it should be blocked properly and impose no impact on blood supply to the spinal cord during surgery.

If simply for discectomy, or carrying out operation between two adjacent vertebral bodies (such as interbody fusion), we do not need the ligation of segmental arteries. As for interbody fusion, we can first insert the disc with the Kirschner wire, identify the target disc by C-arm $\mathrm{X}$-ray machine, then conduct the operation upward and downward around the intervertebral disc (no overtopping the segmental arteries), and carry out discectomy, intervertebral expansion, intervertebral cartilage removal and intervertebral cage placing respectively. If only one vertebral body or two adjacent vertebral bodies fracture without segmental arterial injury, we can also carry out operation in the safe zone without the segmental arteries ligation; while the segmental arteries damage or the screw for anterior spinal osteosynthesis is placed on the equatorial line of vertebral body, we must ligate the segmental arteries. For multi-segment operation, we should conduct the segmental arteries ligation as few as possible. Meanwhile, if the branches of the segmental artery are near the safe zone, we should ligate its branches while the ligation for itself is not needed. If the segmental artery lacks, there is no need concerning the segmental artery protection issue.

Therefore, we believe that the position of safe zone on both sides of thoracolumbar sufficiently ensures that surgeries of this section can be successfully completed under the thoracoscope. With the intervertebral disc (For the intervertebral disc locates well in the middle of the upper and lower segmental artery) as the surgical reference mark, operating up and down separately by the preoperative image data can avoid or reduce injury to blood vessels, and reduce surgical complications. Meanwhile, the differences exist among individuals. Therefore, to make individual surgery plan, preoperative CT imaging should be conducted for the patients; by observing the three-dimensional reconstruction images, the anatomical features and the adjacent relationship of segmental vessels can be got and the distance between the two adjacent segmental arteries should be measured.

When conducting other operation, we also should accurately localize with the $\mathrm{C}$-shape arm X-ray machine to ensure that the operation is conducted on the intervertebral disc level.

\section{Conclusions}

By comparing observation results, we can give specific anatomical parameters to provide a reference for the operation. There are no important blood vessel nerves between the upper and lower segmental arteries on the side of the thoracolumbar spine. The intervertebral disc locates well in this zone, which is the relative safe zone of the blood vessel for anterior approaches. Taking into account the fact that the segmental veins can be ligated without affecting the blood supply, and segmental veins constantly run on the top of the adjacent arteries (or absence), we can take the distance between the two segmental arteries as the maximum range of operation. The segmental arteries arrangement and the adjacent relations can also be clearly displayed by reconstructed CT three-dimensional image. We can find that the arrangement and distribution rules of segmental arteries are basically consistent. Therefore, we can conduct video-assisted thoracoscopic surgery in this zone without damage to the blood vessels, and the threedimensional image can be provided as a reference for surgery plans before operation.

\section{References}

1. Bomback DA, Charles G, Widmann R, Boachie-Adjei O (2007) Video-assisted thoracoscopic surgery compared with thoracotomy: early and late follow-up of radiographical and functional outcome. Spine J 7: 399-405

2. Khoo LT, Beisse R, Potulski M (2002) Thoracoscopic-assisted treatment of thoracic and lumbar fractures: a series of 371 consecutive cases. Neurosurgery 51: S104-117.

3. Schultheiss M, Kinzl L, Claes L, Wilke HJ, Hartwig E (2003) Minimally invasive ventral spondylodesis for thoracolumbar fracture treatment: surgical technique and first clinical outcome. Eur Spine J 12: 618-624

4. Yuan L, Ni GX, Luk KK, Cheung KM, Lu DS, et al. (2005) Effect of segmental artery ligation on the blood supply of the thoracic spinal cord during anterior spinal surgery: a quantitative histomorphological fresh cadaver study. Spine (Phila Pa 1976) 30: 483-486.

5. Li Wei-Guo, Qiu Y, Bin W (2004) Spinal segmental vessel occlusion on the occurrence and development of disc degeneration effects. Journal of Chinese spinal cord 14: 348-351.

6. Rui Bi-yu, Qiu Y, Zhu Yawen (2007) Normal human spinal T5-12 segmental vascular anatomy and imaging study. Anatomic and Clinical 12: 92-94.

7. Orchowski J, Bridwell KH, Lenke LG (2005) Neurological deficit from a purely vascular etiology after unilateral vessel ligation during anterior thoracolumba fusion of the spine. Spine (Phila Pa 1976) 30: 406-410.

8. Koshino T, Murakami G, Morishita K, Mawatari T, Abe T (1999) Does the Adamkiewicz artery originate from the larger segmental arteries? J Thorac Cardiovasc Surg 117: 898-905

9. Mirovsky Y, Hod-Feins R, Agar G, Anekstein Y (2007) Avoiding neurologic complications following ligation of the segmental vessels during anterior instrumentation of the thoracolumbar spine. Spine (Phila Pa 1976) 32: 275-280 